\title{
AN OPEN CLINICAL STUDY TO EVALUATE THE SAFETY AND EFFICACY OF Liv.52 DS IN THE MANAGEMENT OF NON-ALCOHOLIC FATTY LIVER DISEASE (NAFLD)
}

\author{
Gontar Siregar $^{1}$, Rajesh Kumawat ${ }^{2}$, rangesh paramesh ${ }^{2}$, and Palani Dorairaj ${ }^{2}$ \\ ${ }^{1}$ University of Sumatera Utara \\ ${ }^{2}$ Himalaya Drug Company
}

April 28, 2020

\begin{abstract}
Present study was conducted to evaluate the clinical efficacy and safety of Liv.52 DS tablets in the management of Non-Alcoholic Fatty Liver Disease (NAFLD) in an open label clinical study. Sixty male and female patients aged between 18-65 years with NAFLD from clinical examination, laboratory test, and ultrasound findings and those willing to give informed consent were included in the study. At the initial visit, a detailed medical history and symptomatic evaluation was done. Biochemical investigations included total bilirubin, aspartate and alanine amino transferase, serum alkaline phosphatase and total proteins. All the patients received Liv.52 DS at the dose of 2 tablets twice daily for a period of 2 months. Subjects were evaluated at baseline, at the end of 1st month, and at the end of 2ndmonth for Liver function tests, hepatomegaly by ultrasound and NAFLD Score, Lipid profile and for safety assessment of Hematology and biochemical investigations. Non-invasive NAFLD fibrosis score was calculated at each assessment visits to assess the severity of fibrosis due to NAFLD. A subgroup analysis was done in Diabetes Mellitus Subjects. Statistical analysis was carried out using GraphPad Prism, Version 4.03 for windows, Graphpad Software. All the patients completed the study and data was available for analysis. Significant improvement in hepatomegaly, liver function parameters (SGPT, SGOT) was observed. A trend towards improvement in NAFLD score was seen signifying improvement in liver fibrosis due to NAFLD. Safety laboratory investigation results are within normal limits and there were no clinically significant adverse effects were reported during the clinical study. Subgroup analysis carried out in diabetic subjects further demonstrated beneficial effects in those populations suffering from NAFLD with respect to hepatomegaly and LFT levels. From the results of the study, it can be summarised that Liv.52 DS is safe and beneficial in individuals suffering from NAFLD.
\end{abstract}

\section{Hosted file}

Liv.52 DS in NAFLD Indonesia_18.03.2020.doc available at https://authorea.com/users/310018/articles/ 440862-an-open-clinical-study-to-evaluate-the-safety-and-efficacy-of-liv-52-ds-in-the-managementof-non-alcoholic-fatty-liver-disease-nafld 